\title{
Empleo de Sistemas de Información Geográfica en el estudio del Miedo al Delito ${ }^{1}$
}

\author{
Laura Vozmediano Sanz \\ Instituto Vasco de Criminología \\ César San Juan Guillén \\ Instituto Vasco de Criminología
}

\section{RESUMEN}

En el presente trabajo se exploran las posibilidades de los Sistemas de Información Geográfica (SIG) para el estudio de los aspectos espaciales relacionados con el miedo al delito en contextos urbanos, y se presenta un caso concreto: el estudio del miedo al delito en Donostia-San Sebastián. A partir de encuestas a una muestra de residentes, se estudió la distribución del delito objetivo en la ciudad, a partir de datos judiciales, y la distribución del miedo al delito en tres escenarios urbanos distintos. Con estos datos se construyeron los mapas de criminalidad y miedo al delito en la ciudad, comprobando que la percepción subjetiva no era coincidente en el espacio con la criminalidad registrada judicialmente.

Palabras clave: miedo al delito, sistemas de información geográfica.

\section{ABSTRACT}

This work explores Geographic Information Systems' potential for studying some spatial aspects related to fear of crime in urban environments. A particular case is presented: the study of the fear of crime in Donostia-San Sebastián. Crime rates were measure through judicial sentences, and fear of crime was measure through a survey answered by city residents. This data was used to make crime maps and fear of crime maps of the city. Comparing the maps, it was found that the distribution of subjective perception in the city map did not coincide with the distribution of judicially registered crime.

Keywords: fear of crime, geographic information systems

Fecha de recepción: 30-3-2006

Fecha de aceptación: 7-5-2006

\section{Introducción}

\subsection{Los SIG y el estudio del fenómeno delictivo}

La tecnología SIG (Sistemas de Información Geográfica) se emplea para la recopilación, representación y análisis de todo tipo de información referenciada geográficamente. En poco tiempo, este sistema informático se ha instaurado en las prácticas cotidianas de los ámbitos privado, universitario y gubernamental.

Un SIG es una tecnología de manejo de información geográfica por medio de equipos informáticos. Esta información geográfica tiene dos vertientes distintas, la vertiente espacial y la temática (GabrielOrtiz.com, 2006). La espacial hace referencia a los mapas o representaciones cartográficas de enclaves naturales o urbanos, y la temática, a tablas que contienen información alfanumérica. La clave del SIG es que enlaza la información geográfica con la temática, de modo que trabaja al tiempo con una representación geográfica y sus atributos temáticos asociados. Se pueden distinguir varios tipos de programas que, aunque puedan denominarse conjuntamente SIG, tienen diferencias fundamentales en su ámbito de aplicación; entre ellos se encuentra lo que se

\footnotetext{
${ }^{1}$ Agradecimientos: Este trabajo ha sido posible gracias a la financiación del Gobierno Vasco, a través de la concesión a Laura Vozmediano de una beca del Programa de Formación de Investigadores del Departamento de Educación, Universidades e Investigación.
}

Revista Española de Investigación Criminológica

Artículo 2, Número 4 (2006) http://www.criminologia.net 
ha venido a denominarse Desktop Mapping (DM)- sistemas de análisis y visualización integrados entre las aplicaciones Desktop del ordenador personal (Estrada Villegas, 2004). Es un programa de este tipo lo que empleamos en el presente trabajo.

La tecnología SIG pretende ser útil para el estudio y búsqueda de soluciones de problemas del mundo real, trabajando sobre un modelo cartográfico de dicha realidad. Para ello, tiene una serie de funciones que se pueden resumir en las siguientes: Captura y Almacenamiento de datos geográficos y tabulares, Consulta, Análisis y Presentación de los datos, y Resultados presentados en diversos formatos (ESRI, 2004).

Resulta sencillo ejemplificar la aplicabilidad de esta tecnología en el estudio del fenómeno delictivo, ya que su uso se halla muy extendido tanto en el ámbito académico como policial. Un sistema de este tipo nos permitiría construir una base de datos que contenga, por ejemplo, el callejero de una ciudad, datos sociodemográficos de sus habitantes con una referencia espacial (la dirección del censo), datos sobre servicios en cada zona (colegios, bibliotecas, comisarías...) y datos de delitos denunciados con su referencia especial (lugar de comisión del supuesto delito). Bases de datos de este tipo se emplean, en la actualidad, para la elaboración de "mapas del delito", que nos muestran cómo se distribuyen distintos tipos de delitos por la ciudad, y permiten relacionar ese patrón con otras variables. Asimismo, se utilizan para gestionar la respuesta policial en las llamadas de denuncia, o para planificar las necesidades de agentes en las distintas zonas de las ciudades. Por otro lado, es cada vez más usual el empleo de estos sistemas para comunicar a la sociedad la situación en relación a los delitos, y para recibir información de los ciudadanos, a través de Internet. Un ejemplo ilustrativo de este planteamiento lo podemos encontrar en la web www.chicagocrime.org, en la que es accesible todo tipo de información sobre delitos en la ciudad de Chicago, pudiendo realizar búsquedas según el tipo de delito, el emplazamiento... En definitiva, el SIG favorece la comprensión y prevención del delito y ha demostrado su utilidad en muchos países (Weisburd y McEwen, 1997).

En nuestro país su empleo en éste y otros campos es creciente. Stangeland y Garrido de los Santos (2004), por ejemplo, lo emplearon en el análisis de la delincuencia en Málaga, con satisfactorios resultados. Sin embargo, su uso no tiene por qué limitarse al estudio de la delincuencia objetiva, y los mismos autores sugieren la aplicabilidad del sistema para el estudio del miedo al delito, reto que asumimos en este trabajo.

\subsection{Miedo al delito y SIG}

El miedo al delito ha sido ampliamente estudiado por la criminología desde los años 70, pero abordado asimismo desde otras disciplinas, como la sociología, geografía, urbanismo, arquitectura... y también desde la psicología, particularmente la psicología social y ambiental. Esta área de trabajo es inevitablemente multidisciplinar, toda vez que tanto los factores que originan el miedo al delito, como las consecuencias del propio miedo, implican a todas las áreas mencionadas. Para revisiones de la literatura sobre miedo al delito, pueden consultarse trabajos como el de Hale (1996) o recientemente (Miceli, Roccato, \& Rosato, 2004).

En nuestro país, podemos citar importantes contribuciones en al campo del miedo al delito, realizadas desde la psicología social y ambiental. Fernández (1995), Fernández y Corraliza (1996, 1997, 1998), San Juan, Vergara y Germán (2005), Berenguer, Garrido y Montoro (1990), Carro, Valera y Vidal (2005)... realizan interesantes contribuciones a nivel teórico y empírico. En los últimos tiempos, el debate se centra en tres grupos de variables, que pueden configurar las coordenadas de la 
génesis y el mantenimiento del miedo al delito: variables personales, sociales y ambientales.

Las variables personales serían el sexo, edad, capacidades de afrontamiento y control; en definitiva, factores de vulnerabilidad relativa frente al delito. El sexo y la edad son las variables sociodemográficas más comúnmente asociadas con el miedo al delito, si bien la evidencia empírica al respecto es, en ocasiones, contradictoria: muchos estudios afirman que mujeres y ancianos experimentan más temor ante el delito (p.e., Warr, 1984; Skogan y Maxfield, 1981; (Evans \& Fletcher, 2000), pero otros encuentran lo opuesto, o mantienen que esta relación es débil o no existe (Ferraro \& Lagrange, 1992; Chadee \& Ditton, 2003). En cuanto a las capacidades de afrontamiento y control, una mayor percepción de control ante la situación podría reducir el miedo o inseguridad experimentada (Vanderwurff, Vanstaalduinen, \& Stringer, 1989a).

Las variables psicosociales se relacionan con las dinámicas y cohesión vecinales, que podrían ser protectoras frente el miedo al delito, al incrementar el control social. Se han utilizado variables como la satisfacción residencial (Fernández y Corraliza, 1998), apego al barrio (Brown, Perkins, \& Brown, 2003), y sentido de comunidad (Wilson-Doenges, 2000) aunque en algunos trabajos como el de San Juan, Vergara y Germán (2005) estas variables mostraban un escaso poder predictivo frente a posicionamientos más razonados, tales como la confianza en la acción policial y la Justicia.

Finalmente, podemos distinguir dos niveles o clases de variables ambientales relevantes en relación al miedo al delito, y al delito mismo. Por un lado, encontramos trabajos que hacen referencia a la estructura del espacio urbano. Las características estructurales de un lugar podrían inhibir la relaciones sociales, hacerlo más propenso a la ocurrencia de delitos, y provocar más fácilmente miedo al delito; se trataría por tanto de un "espacio crimípeto" (San Juan, 2000). (Vanderwurff, Vanstaalduinen, \& Stringer, 1989b) se refieren al "espacio criminalizable" debido a su características espaciales, e incluyen también el tiempo y la presencia de otros. Por otro lado, hay una fructífera línea de trabajo que se centra en la degradación de los espacios. Destacan aquí la perspectiva de las "actos incívicos" (Lewis y Maxfield, 1980) y la teoría de las "ventanas rotas" (Wilson y Kelling, 1982). Un espacio con signos de degradación indicaría un control social debilitado, produciendo más deterioro, miedo al delito en aumento, evitación de ese espacio, y finalmente un aumento del delito. Desde esta perspectiva, un reciente e interesante trabajo de Doran y Lees (2005) introduce precisamente los SIG para el estudio de la relación entre el desorden, el delito y el miedo al delito. Tanto las variables relacionadas con la estructura, como las relacionadas con la degradación de los espacios pueden combinarse a la hora de estudiar su influencia en el tema que nos ocupa; así lo hace, por ejemplo Fernández (1985) en su trabajo sobre "lugares peligrosos". Los tres grupos de variables mencionadas (personales, sociales y ambientales) configuran la perspectiva psico-socioambiental desde la que vamos a abordar el análisis del miedo al delito.

Centrándonos en esta ocasión en la relevancia de las variables ambientales, la hipotetizada influencia de las características de los espacios en el miedo a ser víctima de un delito, debería originar una desigual distribución de esta variable en los distintos escenarios de la ciudad. Es decir, en la representación cartográfica de una urbe concreta, la distribución del miedo no sería homogénea. Aún cuando se encuentre esta distribución no uniforme, para poder afirmar que la perspectiva psico-socio-ambiental es relevante, es necesario establecer que dicha distribución desigual del miedo al delito no se debe a hipótesis rivales: la distribución del delito registrado judicialmente, y la distribución de la victimación, serían dos de ellas. Para comprobar si estas dos 
cuestiones se cumplen en un escenario concreto, el equipo consideró que el empleo de SIG sería de gran utilidad, además de constituir un reto que, de superarse con éxito, podría emplearse en investigaciones futuras.

Por todo ello, el objetivo del presente trabajo fue construir un mapa del delito en la ciudad de San Sebastián, junto con mapas temáticos de la victimación y el miedo al delito, con objeto de contrastar la distribución de estas variables.

\section{Método}

\subsection{Muestra}

La muestra estuvo compuesta por 504 sujetos residentes en San Sebastián, distribuidos en tres grupos con igual número de integrantes, y residiendo cada grupo en uno de los escenarios que componen el estudio. Estos escenarios fueron 3 barrios de la ciudad, concretamente los denominados Centro, Amara y Alza, que fueron seleccionados como prototípicos de tres diferentes niveles socio-económicos, alto, medio y bajo respectivamente. El género estuvo equilibrado al $50 \%$, estableciéndose la media de edad en los 48 años.

Adicionalmente, y con intención de comparar el miedo al delito con la distribución de la delincuencia objetiva en la ciudad, se complementó con otra investigación también desarrollada por el Instituto Vasco de Criminología, que consistió en un estudio sobre la criminalidad registrada judicialmente en Guipúzcoa, en el cual se recogió una muestra de 869 sentencias. Para los propósitos de este trabajo, se tomaron en cuenta todas las sentencias referidas a delitos ocurridos en la ciudad de Donostia-San Sebastián, 311 en total.

\subsection{Materiales y procedimiento}

El presente trabajo se enmarca en un estudio más amplio, para la creación, validación y fiabilización de un cuestionario para la evaluación de la percepción de inseguridad y miedo al delito en la C.A.V., en el cual se entrevistó en sus domicilios a 1512 personas de las tres capitales de esta comunidad autónoma. Para más información sobre algunos aspectos de dicho trabajo, ver San Juan, Vergara y Germán (2005). Se empleó un cuestionario elaborado ad hoc, que presentó un perfil psicométrico aceptable. De entre sus escalas, en el presente estudio se ha trabajado con las siguientes:

- Escala de miedo al delito: formada por 5 ítems, en los que se recoge el grado de gravedad de la inseguridad ciudadana en su barrio, grado de preocupación que le genera la inseguridad ciudadana en su vida cotidiana, miedo a ser víctima de un atraco o agresión en la calle, miedo a ser víctima de un robo en el domicilio, y probabilidad estimada de ser víctima de un delito grave o preocupante (respuesta de 0 a 10 para cada ítem).

- Victimación: en caso de haber sido víctima de algún delito, grado de impacto emocional que le ha causado (de 0 a 10).

- Satisfacción con los vecinos: grado de satisfacción con sus vecinos (de 0 a 10).

- Satisfacción con el entorno físico: integrado por 4 ítems que hacen referencia a los parques y zonas ajardinadas, zonas peatonales, servicio de limpieza, y seguridad y vigilancia, respondiendo de 0 a 10 .

- Inseguridad relativa: nivel de inseguridad del barrio en comparación con otros de la ciudad (de 1 a 3 ). 
- Jueces: grado de satisfacción con la actuación de los jueces frente a la inseguridad ciudadana (de 0 a 10$)$.

- Ayuntamiento: Satisfacción con la política de seguridad del ayuntamiento (de 0 a 10).

Respecto a los datos sobre delincuencia, se tomaron, como se ha dicho, de un estudio sobre la delincuencia registrada en la última década en la Audiencia Provincial de Guipúzcoa En dicho estudio, trabajando con una cifra aproximada de más de veinticinco mil sentencias como universo de la investigación, se utilizó un muestreo obtenido por azar sistemático. Se eligió al azar una primera unidad de la muestra, inferior al coeficiente de elevación, fijado en veintiocho. Las restantes sentencias analizadas se seleccionaron sumando, al número de expediente de dicha unidad, el coeficiente de elevación, de forma sucesiva, hasta obtener todos los expedientes necesarios para la muestra (margen de error del cinco por cien; margen de confianza del 99,7\%). Empleando este procedimiento, la muestra quedó integrada por 869 sentencias. De ellas, se tomaron para este trabajo las 311 correspondientes a la ciudad objeto de interés. El dato concreto que nos interesó fue la localización de los delitos, que estaba recogido en forma de código postal en todos los casos.

En cuanto al marco espacial del trabajo, la ciudad de Donostia-San Sebastián, se empleó un mapa digital que el Gobierno Vasco pone a disposición de los ciudadanos en su página web. Correos proporcionó un mapa en papel con los límites de los códigos postales, a partir del cual se construyó un mapa digital con esta zonificación. La combinación de estos mapas con los datos de las encuestas y sentencias, en el programa ArcGIS 9.0, permitió elaborar los mapas temáticos que fueron analizados en el estudio.

\subsection{Diseño}

Se empleó un diseño de encuesta, siguiendo un procedimiento estratificado y polietápico por conglomerados en cuanto a la selección de los escenarios donde realizar las encuestas. En cada uno de los escenarios, la selección de las personas a entrevistar en su domicilio se realizó siguiendo rutas aleatorias, a partir de una dirección de partida establecida de antemano, y cumpliendo cuotas de sexo, edad y actividad.

\section{Resultados}

El primer resultado del proceso descrito fue un mapa de densidad de delito registrado judicialmente para cada código postal de la ciudad (Figura 1). En el SIG, cada una de las parcelas que representa un código postal, tenía su valor asociado en la variable "número de delitos"; este número, dividido por la extensión de la parcela, nos proporciona el índice de densidad de delito representado en el mapa, que permite establecer comparaciones entre parcelas a pesar de la disparidad de área de cada una de ellas.

Este mapa temático proporcionó un marco de comparación con los datos de delincuencia, sobre el cual representar los datos de miedo al delito y victimación. El segundo mapa realizado contenía las zonas referidas a los tres niveles socioeconómicos. Para cada escenario, se codificó en el SIG los valores que le correspondían en las variables contempladas en el cuestionario. Estos tres escenarios, al superponerlos con el mapa de la figura 1, posibilitaron comparar la distribución de estas variables con el delito registrado judicialmente. En este punto es necesario indicar que, en todas las representaciones que se ofrecen a continuación, las diferencias en colores o en niveles implican diferencias estadísticamente significativas. 


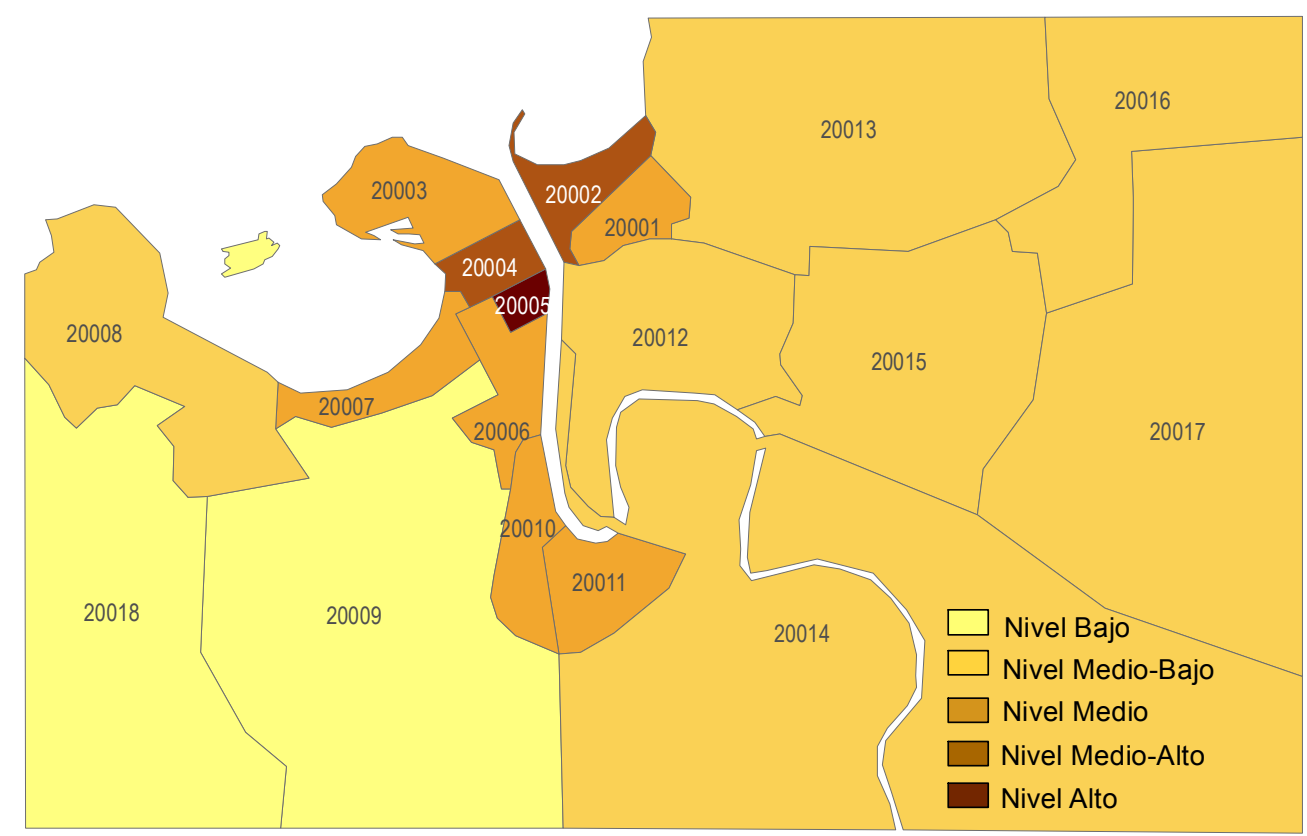

Figura 1. Densidad de delito en Donostia -San Sebastián.

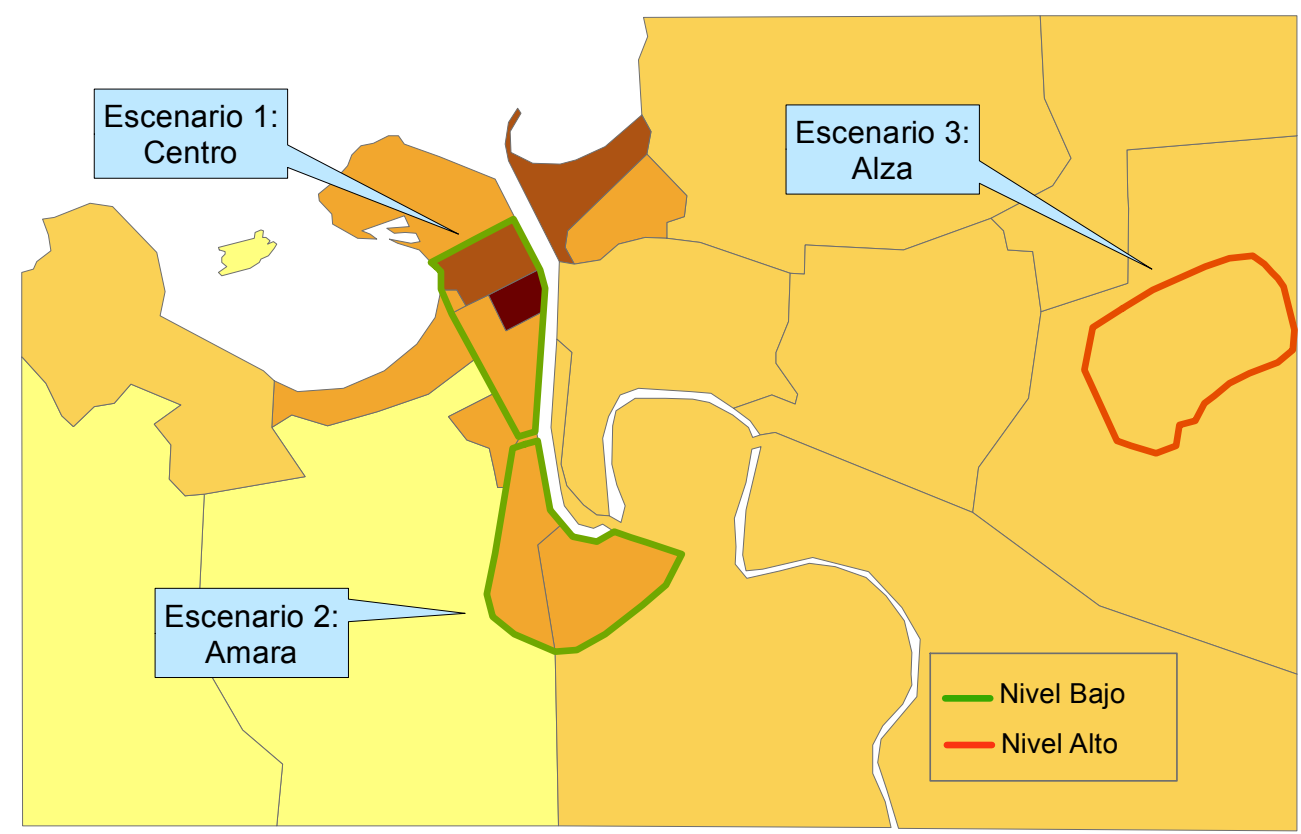

Figura 2. Miedo al delito en los tres escenarios escogidos para el estudio.

En la figura 2 se presenta el miedo al delito en los tres escenarios, superpuesto al mapa inicial de densidad del delito. Se puede observar que los residentes en uno de los tres escenarios, reportan un miedo al delito mayor que el resto de sujetos. Sin embargo, ese escenario no se ubica en la zona con mayor densidad de delito; al contrario, los otros dos escenarios soportan una densidad mayor.

Revista Española de Investigación Criminológica

Artículo 2, Número 4 (2006) http://www.criminologia.net 
En cuanto a la magnitud del miedo en cada escenario, la media de miedo en el escenario 1 fue de 25,18, en tanto que en el escenario 2 fue de 26,46. En el escenario 3, donde se encuentra un miedo significativamente mayor que en los anteriores, la media de miedo al delito fue de 28,75. Al ser la escala de 50 puntos, en todos los casos la puntuación se situó por encima del punto medio.

En la figura 3, se muestra el resultado de la superposición de los datos de victimación de los residentes en los tres escenarios, sobre el mapa de delito registrado judicialmente. En este caso, las diferencias estadísticamente significativas entre escenarios son coherentes con la distribución de la densidad de delito en la ciudad.

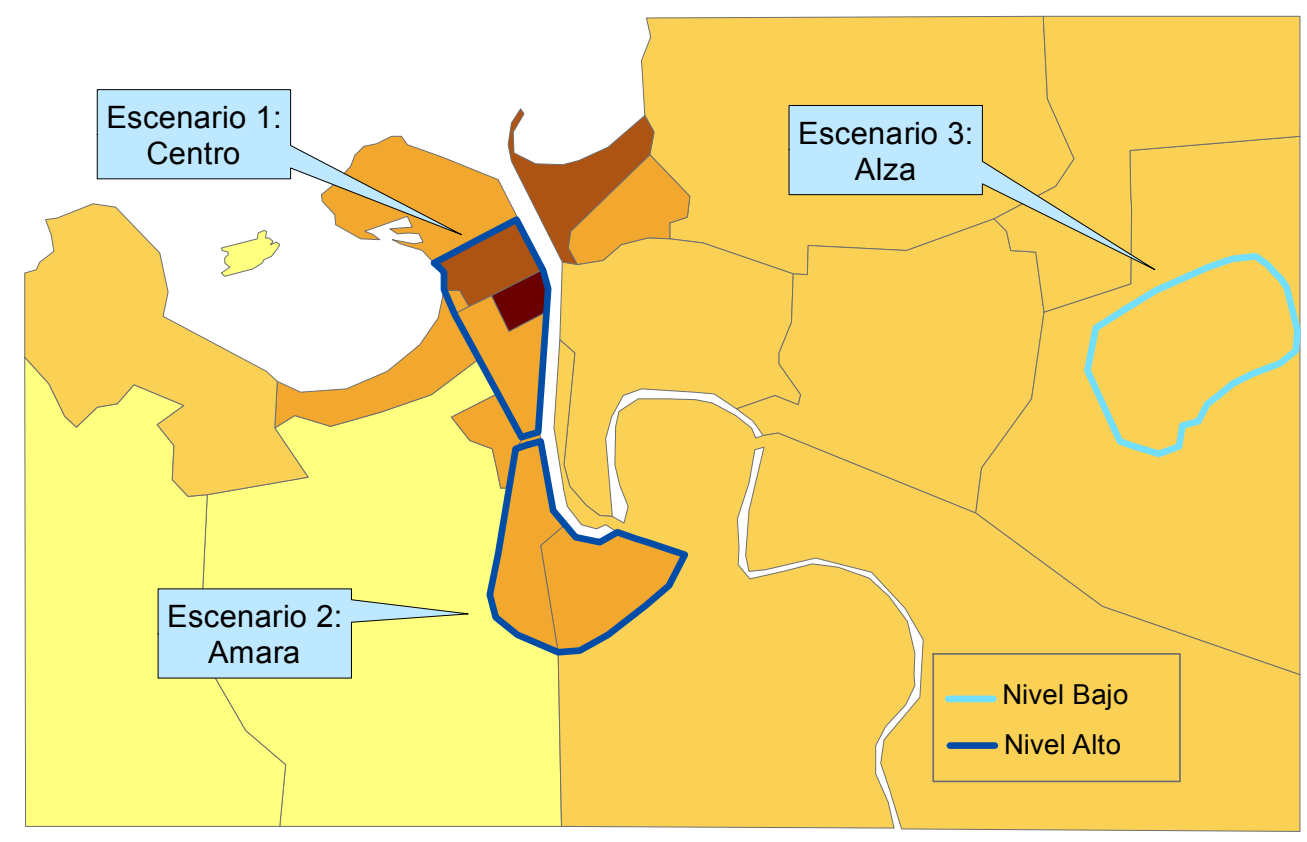

Figura 3. Niveles de victimación en los tres escenarios.

Tomando en cuenta el miedo al delito superior en el escenario 3, no explicado por la densidad de delito y la victimación, se exploró la distribución en los tres barrios de las variables psico-socio-ambientales recogidas en el cuestionario: satisfacción con el espacio físico, satisfacción con los vecinos, inseguridad relativa, satisfacción con actuación de los jueces frente a la inseguridad ciudadana y satisfacción con la política de seguridad del ayuntamiento. Se encontró que los residentes en el escenario 3 mostraron: menor satisfacción con el espacio físico que los otros dos escenarios; menor satisfacción con los vecinos que el escenario 1, mayor percepción de su barrio como inseguro, en comparación con otros, que los otros dos escenarios; y menor satisfacción con las actuaciones judiciales y con la política del ayuntamiento que los otros dos escenarios. Todas estas diferencias fueron estadísticamente significativas con al menos $\mathrm{p}<0.05$.

\section{Discusión}

La no coincidencia entre los espacios que soportan mayor densidad de delito -y mayor victimación- con el espacio en el que los residentes reportan un miedo al delito superior se puede interpretar en términos de la denominada paradoja del miedo al delito (Fattah, 1993). Se trata de un fenómeno frecuente pero, en todo caso, no absolutamente 
generalizable, ya que los resultados son en ocasiones contradictorios, poniendo posiblemente en evidencia la importancia de la validez ecológica de este tipo de investigaciones. En nuestro estudio existe, en todo caso, una correlación negativa entre delincuencia objetiva y miedo al delito subjetivo que merece ser tenida en cuenta a la hora de explicar el fenómeno de la percepción de inseguridad desde una perspectiva psico-socio-ambiental. Dicha perspectiva podría incluso explicar la disparidad de resultados mencionada. La influencia de las características de los espacios dibujaría en cada contexto urbano estudiado un patrón de miedo al delito propio, coincidente, en unos casos con el delito objetivo, pero no en otros. Otro elemento que puede influir en la disparidad de resultados al respecto es el desplazamiento del fenómeno delictivo en la ciudad. Desde una perspectiva espacio-temporal, una zona que ha soportado altos índices de delito en el pasado, podría mantener un mayor nivel de miedo al delito aún cuando el delito haya "migrado" a otra zona de la ciudad como consecuencia, por ejemplo, de la intensificación puntual de la acción policial. La percepción de ese espacio como peligroso podría perdurar más allá de elementos objetivos, una vez que se ha incorporado a las dinámicas sociales de la vida cotidiana en un vecindario concreto.

Por otro lado, y frente a la no relación del miedo al delito con los datos de criminalidad objetiva, las variables psico-socio-ambientales incluidas en el estudio sugieren que la percepción del espacio físico y los vecinos, así como la creencias compartidas sobre la efectividad de la justicia y el papel del ayuntamiento en garantizar la seguridad, podrían estar jugando un papel en la génesis y mantenimiento del miedo al delito. Claro que los factores estructurales del espacio son difícilmente aislables en la investigación. Es evidente que las configuraciones urbanísticas con una visión más funcional, o que no respondan a patrones de tipo estético, suelen ser el escenario de convivencia de las clases más desfavorecidas, como es el caso de los suburbios de muchas grandes ciudades europeas convertidos en guetos para población inmigrante que, antes de sufrir cualquier proceso de degradación o vandalismo, no respondían en un inicio a parámetro alguno de calidad de vida urbana. En este caso las diferencias obtenidas en la percepción de miedo pueden estar condicionadas por el estatus socioeconómico antes que por elementos de configuración del espacio: Así, parece que en nuestro contexto el estatus bajo reporta mayor miedo, como ocurriría en Estados Unidos, al contrario que en América Latina, donde los más ricos reportan mayores sentimientos de inseguridad (Gaviria y Vélez, 2001).

Tratándose de un estudio exploratorio, que busca abrir una línea de trabajo, no cabe hacer afirmaciones tajantes, pero nuestros resultados sugieren la pertinencia de seguir investigando la influencia de estas variables en la percepción de inseguridad, en general, y en la valoración de la probabilidad de ser víctima de un delito, en particular.

En todo caso, las diferentes aproximaciones existentes en relación a la medición del miedo al delito pueden explicar parcialmente la disparidad de resultados. En este sentido, Farral y Gadd (2004) señalan que usualmente se pregunta por la intensidad del miedo al delito, y muy raramente por su frecuencia, lo que produce sobre-estimación en los resultados. En su estudio incluyen ambos tipos de medidas y preguntan por los episodios de miedo en el último año, concluyendo que en el Reino Unido, la exposición habitual a altos niveles de miedo no es muy común. Jackson (2005) retoma este tema, planteando que la causa de la sobre-estimación puede ser el hecho de que los sujetos recuerden la más intensa y llamativa de las experiencias, e infieran que ese nivel de miedo es representativo de sus experiencias. Apuesta por preguntar la frecuencia de episodios de preocupación por ser víctima de ciertos delitos, en el plazo del último mes.

Respecto a la consistencia de los indicadores de delincuencia objetiva, cabe hacer una serie de consideraciones. A la hora de analizar el fenómeno criminal, las 
cuatro fuentes de datos quizás más habituales son las encuestas de victimación, los datos judiciales, los datos policiales y, finalmente, los datos penitenciarios. Cada una de estas fuentes tiene sus virtudes y sus limitaciones. Las encuestas de victimación tienen, evidentemente, las limitaciones propias de las medidas de autoinforme; los datos obtenidos de sentencias judiciales, por su parte, esconden una muy comentada "cifra negra" de delitos que no llegan hasta el sistema judicial. Pero en ambos casos, la información que proporcionan puede ser más rica y completa que la que se obtendría por medio de bases de datos policiales o penitenciarios, que tampoco están exentas de fuentes de error, como por ejemplo, las denuncias falsas o el sesgo clasista de la población penitenciaria. En cualquier caso, al contemplar dos de las posibles fuentes de datos, entendemos que el retrato de la distribución geográfica del delito en DonostiaSan Sebastián queda adecuadamente perfilado. Además, cabe señalar que el mapa obtenido es coincidente con los datos policiales que se hacen públicos periódicamente, aunque estos últimos no se contemplen de modo específico en el trabajo.

Junto al análisis de la distribución del miedo al delito en una urbe concreta, era objetivo de este trabajo la evaluación del empleo de software SIG para este campo de estudio. La experiencia ha resultado positiva, toda vez que nos ha posibilitado construir el mapa del delito y del miedo al delito en tres escenarios diferentes de un mismo contexto urbano. La explotación de datos con este software tiene una ventaja adicional muy pertinente desde el punto de vista de la comunicación, ya que se pueden presentar los resultados mediante sucesivas representaciones cartográficas temáticas. En este sentido, puede ser particularmente importante su aplicabilidad para el asesoramiento en la toma de decisiones sobre políticas de seguridad. Un tercer elemento positivo es la amplitud de posibilidades que ofrece el empleo de esta herramienta para la exploración de nuevas ideas y contrastación de hipótesis. Cualquier otra información referenciada geográficamente, que pueda tener relevancia, es susceptible de ser incluida en el estudio. En este caso, por ejemplo, se podrían incluir la localización de pasos subterráneos y de parques, localización de comisarías, número de efectivos policiales asignados a cada área, diferenciar tipologías delictivas, etc. Cabe decir que la principal limitación para su aplicación consiste en la necesidad de disponer de una cartografía en formato digital de referencia del escenario geográfico objeto de estudio que sea compatible con el programa. Por otra parte, sería necesario hacer algunos ajustes en los datos de frecuencia de delitos registrados que se reflejan en la representación cartográfica, ya que son simples indicadores de prevalencia que no contemplan la ratio por densidad de población o grado de urbanización. Al margen de estas limitaciones, podemos afirmar que este estudio exploratorio consigue poner de manifiesto la relevancia de los escenarios y sus características sociales y ambientales en el miedo al delito urbano, y lo hace utilizando una herramienta que produce representaciones amigables, que pueden ser fácilmente comprendidas y empleadas para la toma de decisiones. Además, las representaciones espaciales de los datos favorecen nuevos caminos para la reflexión y el descubrimiento de relaciones entre las variables.

Pensamos, por tanto, que el empleo de SIG en el futuro permitirá abordar una serie de cuestiones que permanecen sin resolver, en relación al miedo al delito. Concretamente, es necesario determinar qué variables psico-socio-ambientales, y en qué medida, explican la génesis y el mantenimiento de este miedo. Además, sería necesario introducir la dimensión temporal en los estudios, para abordar la evolución del fenómeno en el espacio y en el tiempo. Habría que estudiar, asimismo, la distribución del miedo al delito en una serie de ciudades distintas, lo que nos ofrecería la posibilidad de comparar sus patrones. Y por último, resulta necesario estudiar el miedo a distintos 
tipos de delitos de modo independiente, para determinar su frecuencia y su distribución espacial.

\section{Bibliografía.}

Berenguer, R., Garrido V. y Montoro, L. (1990). El miedo al delito en Valencia. Un estudio psicosocial. Delincuencia / Delinquency, 2:169-184.

Brown, B., Perkins, D. D., \& Brown, G. (2003). Place attachment in a revitalizing neighborhood: Individual and block levels of analysis. Journal of Environmental Psychology, 23: 259-271.

Carro, D., Valera, S. y Vidal, T. (2005). Inseguridad percibida en el espacio público: variables personales, sociales y ambientales en un estudio de un barrio de Barcelona. En R. García Mira, A. Fernández González, M. D. Losada Otero y M. Golugoff Scheps (Comps.). Psicología Ambiental, Comunitaria y de la Educación. Madrid: Biblioteca Nueva.

Chadee, D. \& Ditton, J. (2003). Are older people most afraid of crime? Revisiting Ferraro and LaGrange in Trinidad. British Journal of Criminology, 43: 417-433.

Doran, B. J. \& Lees, B. G. (2005). Investigating the spatiotemporal links between disorder, crime, and the fear of crime. Professional Geographer, 57: 1-12.

ESRI (2004). Introducción a ArcGIS I. Material inédito, entregado en el curso "Introducción a ArcGIS I y II" impartido por ESRI España Geosistemas.

Estrada Villegas, J. (2004). Los Sistemas de Información Geográfica y la Seguridad Pública. [Disponible en línea]. Portal Belt Ibérica S.A. Analistas de Prevención: http://www.belt.es/expertos/experto.asp?id=2229

Evans, D. J. \& Fletcher, M. (2000). Fear of crime: testing alternative hypotheses. Applied Geography, 20: 395-411.

Fernández Ramírez, B. y Corraliza, J. A. (1996). Aspectos físicos y sociales en los lugares peligrosos. Miedo al delito en un espacio institucional. Revista de Psicología Social, 11(2): 219-234.

Fernández Ramírez, B. y Corraliza, J. A. (1997). Hacia una tipología de lugares peligrosos, en relación con el miedo al delito. Intervención Psicosocial, 6: 237248.

Fernández Ramírez, B. y Corraliza, J. A. (1998), Generalidad y especificidad en la explicación del miedo al delito. Apuntes de Psicología, 16 (1-2): 173-186.

Fernández Ramírez, B. (1995). Lugares peligrosos. Psicología Ambiental y miedo al delito. Colección Tesis en Microficha. Servicio de Publicaciones: Universidad Autónoma de Madrid.

Ferraro, K. F. \& Lagrange, R. L. (1992). Are Older-People Most Afraid of Crime Reconsidering Age-Differences in Fear of Victimization. Journal of Gerontology, 47: S233-S244.

GabrielOrtiz.com (2006).Qué son los Sistemas de Información Geográfica. Tipos de SIG y modelos de datos. Un artículo introductorio para entender las bases de los SIG. [Disponible en línea]. http://recursos.gabrielortiz.com/index.asp?Info=012

Gaviria, A. y Vélez, C. E. (2001). Who Bears the Burden of Crime in Colombia? [Disponible en línea] ftp://ftp.fedesarrollo.org.co/pub/documentos/WP23.pdf

Hale, C. (1996). Fear of crime: a review of the literature. International review of Victimology, 4: 79-150.

Lewis, D. y Maxfield, M. (1980). Fear in the Neighbourhoods: An investigation of the impact of crime. Journal of Research in Crime and Delinquency, 17: 160-89. 
Miceli, R., Roccato, M., \& Rosato, R. (2004). Fear of crime in Italy - Spread and determinants. Environment and Behavior, 36: 776-789.

San Juan, C. (2000): Theories of design and designs of theory in the environmental interventions. Bulletin of People Environment Studies, 15: 15-16.

San Juan, C., Vergara, A. y Germán, I. (2005). Propiedades psicométricas de un cuestionario para la evaluación de la calidad de vida urbana y el miedo al delito. Revista Española de Investigación Criminológica, 3: 1-13.

http://www.criminologia.net/Revista.htm

Skogan, W. and M. Maxfield. (1981). Coping With Crime. Beverly Hills, CA: Sage Publications.

Stangeland, P. y Garrido de los santos, M. J. (2004). El mapa del crimen. Valencia: Tirant lo blanch.

Vanderwurff, A., Vanstaalduinen, L., \& Stringer, P. (1989). Fear of Crime in Residential Environments - Testing A Social Psychological Model. Journal of Social Psychology, 129: 141-160.

Warr, M. (1984). Fear of victimization: Why are women and the elderly more afraid. Social Science Quaterly, 65: 681-702.

Weisburd, D. y McEwen, T. (1997). Crime Mapping and Crime Prevention. Monsey, New York: Criminal Justice Press.

Wilson, J. Q. y Kelling, G. L. (1982). The police and neighborhood safety: Broken windows. The atlantic Monthly, 249: 29-38.

Wilson-Doenges, G. (2000). An exploration of sense of community and fear of crime in gated communities. Environment and Behavior, 32: 597-611.

\section{LOS AUTORES}

Laura Vozmediano es licenciada en psicología por la Universidad del País Vasco y becaria del Programa de Formación de Investigadores del Departamento de Educación, Universidades e Investigación del Gobierno Vasco, en el Instituto Vasco de Criminología. Sus áreas de interés son la ecología del delito y el miedo al delito en escenarios urbanos.

César San Juan es Doctor en Psicología Social por la Universidad Católica de Lovaina (Bélgica). Es subdirector de Investigación del Instituto Vasco de Criminología donde desarrolla las siguientes líneas de investigación: Política criminal y prácticas penales, Delincuencia juvenil y de menores, Ecología del delito, Percepción de inseguridad, miedo al delito y prevención de la delincuencia, Ciencia policial y forense, y Victimología y justicia restaurativa. Dirige el Centro Internacional de Investigación sobre la Delincuencia, la Marginalidad y las Relaciones sociales (DMS International Research Centre) impulsado por la Sociedad Internacional de Criminología. 